\title{
Providing an acute oncology service during the COVID-19 pandemic
}

\author{
Authors: Shanthini M Crusz, ${ }^{A *}$ Peter E Hall, ${ }^{A *}$ Kate Earwicker, ${ }^{B}$ Sue Dexter, ${ }^{B}$ Gemma Patel-Walker, ${ }^{B}$ Thomas Powles ${ }^{A}$ \\ and Nikolaos Diamantis ${ }^{A}$
}

The COVID-19 pandemic has led to adaptations being made to all aspects of the NHS, including general practice, acute medical specialties and oncology. This has presented unique challenges to acute oncology services (AOSs) in how to provide continuity of care while maintaining the safety of patients and staff. We describe the experience of the AOS team at Barts Health NHS Trust, working across three acute hospitals in east London. Changes to the service due to COVID-19 included increased remote reviews and referrals to the specialist oncology cancer acute assessment unit. The patient population reviewed in April 2020 (at the initial peak of the pandemic in the UK) was markedly different to one reviewed in April 2019, with 55\% more patients presenting with a new diagnosis of cancer via an emergency route. Finally, we suggest changes to AOSs for future waves of the pandemic.

KEYWORDS: cancer services, coronavirus, pandemic, SARS-CoV-2, systemic anti-cancer therapy

DOI: $10.7861 /$ clinmed.2020-0693

\section{Introduction}

Studies have identified several independent factors associated with increased mortality from COVID-19, including older age, being male, history of smoking, the presence of comorbidities and active cancer., ${ }^{1,2}$ In March 2020, the UK introduced an initial lockdown as a way to 'flatten the peak' of the pandemic and thus limit the impact on the NHS. ${ }^{3}$ This led to the reduction or cessation of services not directly related to the care of patients with COVID-19, including 2-week-wait referral pathways for suspected cancer and most cancer treatments. ${ }^{4}$ Barts Health NHS Trust is one of the largest NHS trusts in the UK, covering a population of 2.5 million in east London in an area characterised by significant diversity and health inequalities. The acute oncology service (AOS) was established in the response to the National Confidential Enquiry into Patient Outcomes and Death (NCEPOD) and National Chemotherapy Advisory Group (NCAG) reports and provides support to all cancer patients who

Authors: ${ }^{A}$ consultant medical oncologist, Barts Health NHS Trust, London, UK; ${ }^{B}$ acute oncology nurse, Barts Health NHS Trust, London, UK; *joint first authors are admitted with acute complications from their cancer or treatment complications, and patients in whom a first diagnosis is suspected. ${ }^{5,6}$ This rapid report compares the characteristics of the patients reviewed by our AOS during the initial COVID-19 peak in April 2020 with those of patients admitted in April 2019 and discusses how AOS at Barts Health was adapted to meet the challenge posed by COVID-19, with implications for future waves of the pandemic.

\section{Methods}

Data were retrospectively collected and analysed for all patients with a known or suspected cancer diagnosis who presented via the emergency department (ED), were admitted and underwent next day AOS assessment within three Barts Health NHS Trust acute hospitals (Royal London Hospital, Newham University Hospital and Whipps Cross University Hospital) from 1-30 April 2020 and compared with data from the same period in 2019. Each patient was counted only once per admission even if they received multiple AOS reviews during that admission. Patient details were derived from the AOS admissions database and demographic/admission details were collected retrospectively using the Trust's electronic patient record system. The scope and method of data collection were the same for 2019 and 2020. The reason for admission was classified into one of four categories according to the Royal College of Physicians Acute Oncology toolkit - 1: new cancer diagnosis; 2: complications of cancer treatment; $3 \mathrm{~A}$ : acutely unwell from disease itself; 3B: acutely unwell from other comorbidity. This audit was registered and approved within the Trust (ref. 11242).

\section{Results}

The total number of patients requiring AOS review was 141 in April 2020 compared to 209 in April 2019, a decrease of 32.5\% (Fig 1). This represents 198 individual patients in 2019, with nine patients admitted twice and one patient admitted three times. In 2020, this represents 137 individual patients, with four patients admitted twice. Total patient demographics are summarised in Table 1. There was a shift in the pattern of tumour types presenting, notably in breast cancer patients, where numbers presenting reduced from 31 (15.7\% of total) in 2019 to 7 (5.1\%) in 2020. In contrast, the number of prostate cancer patients rose from 15 (7.6\%) in 2019 to 20 (14.6\%) in 2020, which is likely to reflect the known risk factors for COVID-19, namely being older, male and having active cancer (Fig 1)., ${ }^{1,8}$ 


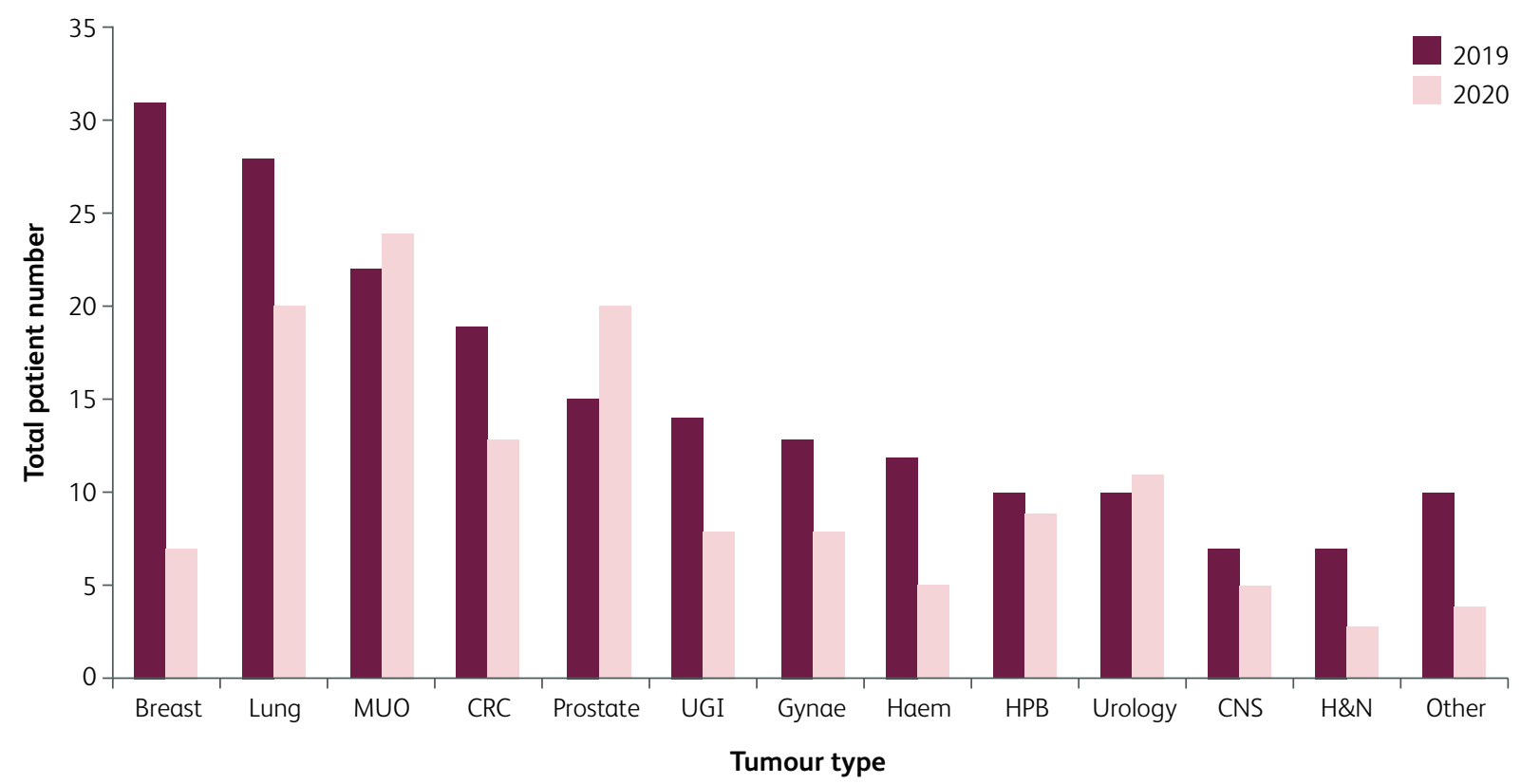

Fig 1. Primary tumour type. $C R C=$ colorectal cancer; $C N S=$ central nervous system; $H \& N=$ head and neck; HPB = hepatobiliary; $M U O=$ malignancy of unknown origin; UGI = upper gastrointestinal.

The reasons for admission are shown in Fig 2. There was a 55.0\% increase in the number of patients presenting with a new cancer diagnosis in April 2020 compared to April 2019. The number of patients admitted with a complication of their cancer treatment fell by $86.9 \%$, whereas the number admitted who were acutely unwell from other comorbidities rose by $145 \%$. Interestingly, the number of patients who presented as a result of being acutely unwell due to their cancer decreased by $50.9 \%$. In 2019, $85.2 \%$ of patients (178) survived to discharge with $14.8 \%$ (31 patients) dying. In $2020,77.3 \%$ of patients were discharged and $22.0 \%$ (31 patients) died.

Escalation plans were examined to see if COVID-19 had led to alterations in practice. In April 2019, 37\% of patients had do not attempt cardiopulmonary resuscitation (DNACPR) orders signed, compared to $67 \%$ in April 2020. This is likely due to the increased need for clear escalation plans during COVID-19 to assist in allocation of limited critical care resources. No AOS patient was admitted to the intensive care unit (ITU) in 2019, whereas four were admitted in 2020.

In April 2020, 108 AOS patients underwent SARS-CoV-2 antigen testing during their admission, with 32 patients (30\%)

Table 1. Patient demographics

$\begin{array}{lll} & \text { April 2019 } & \text { April 2020 } \\ \text { Patient reviews, } \mathrm{n} & 209 & 141 \\ \text { Median age, years (range) } & 65(25-100) & 73(32-98) \\ \text { Male sex, } \mathrm{n}(\%) & 90(43) & 82(58) \\ \text { Ethnicity } & & \\ \text { Non-white, } \mathrm{n}(\%) & 78(37) & 55(39) \\ \text { Not stated, } \mathrm{n}(\%) & 5(2) & 2(1)\end{array}$

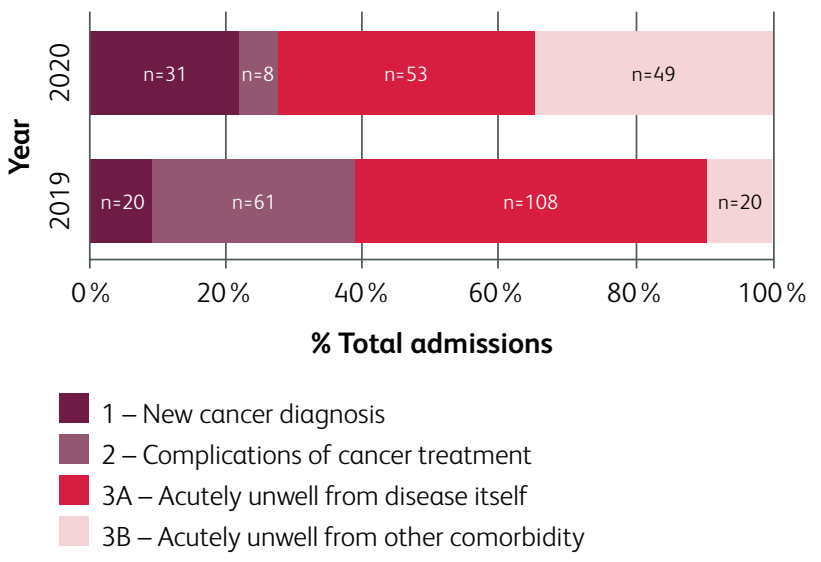

Fig 2. Types of AOS admissions in April 2019 compared to April 2020.

returning a positive result. A comparison of the demographics and characteristics for the negative/not tested patients versus the positive patients is shown in Table 2 . Of the patients with prostate cancer, $52.4 \%(11 / 21)$ tested positive compared to $14.3 \%(1 / 7)$ with breast cancer. The ceiling of care was ward-based (not for resuscitation) for 28 patients ( $87.5 \%$ ), non-invasive ventilation (NIV) for two patients (6.3\%) and ITU for two patients $(6.3 \%)$. Neither patient admitted to the ITU (one for intubation, one for NIV) had active cancer, having undergone radical treatment for prostate cancer over one year previously. Fifteen (46.9\%) of the patients who were positive died.

Adaptations were made to the AOS in order to reduce the risk of coronavirus transmission to patients and staff. There was a $94 \%$ reduction in the number of face-to-face reviews, with the majority occurring remotely instead. Similarly, the chemotherapy hotline, 
Table 2. Demographics and characteristics of patients with a positive test for SARS-CoV-2 compared with those with a negative test or were not tested

$\begin{array}{lll} & \begin{array}{l}\text { Negative/not } \\ \text { tested }(\mathbf{n}=\mathbf{1 0 9})\end{array} & \begin{array}{l}\text { Positive } \\ (\mathbf{n}=\mathbf{3 2})\end{array} \\ \text { Median age, years (range) } & 72.8(32-93) & 76.1(52-98) \\ \text { Male, } \mathrm{n}(\%) & 57(52.3) & 25(78.1) \\ \text { Non-white ethnicity, } \mathrm{n}(\%) & 40(36.7) & 19(59.4) \\ \text { SACT within 30 days of } & 26(23.9) & 8(25.0) \\ \text { admission, } \mathrm{n}(\%) & & 15(46.9) \\ \text { Died, } \mathrm{n}(\%) & 16(14.7) & \end{array}$

SACT $=$ systemic anti-cancer therapy.

a 24-hour service that is the first point of contact for oncology patients who are unwell at home, issued more telephone advice in April 2020 compared to April 2019 (Table 3). If face-to-face assessment of a patient was required, the hotline assessor would assess and discuss with the patient the potential places for medical review, which included their local ED, GP or the cancer acute assessment unit (CAAU), a specialist unit run by oncologists at St Bartholomew's Hospital. After discussion with the patient the decided destination of referral was recorded (Table 3). Interestingly, the chemotherapy hotline in April 2020 preferentially referred patients to the CAAU, rather than to their GP or to the ED. We speculate that factors contributing to this change in medical review included a perceived higher risk of exposure to COVID in local EDs (which were previously more convenient for patients to attend compared to the central location of St Bartholomew's Hospital CAAU) and decreased face-to-face reviews by GP services. It is important to note that patients on cancer treatment also accessed emergency treatment via the ED independently of the hotline services, for example by self-referral, calling 999 or on the advice of a cancer nurse specialist; thus data from the hotline services does not extrapolate to the overall AOS population (Fig 2).

\section{Discussion}

Our findings indicate changes in the AOS population during the initial peak of the COVID-19 pandemic, with implications for service planning and patient care. Specifically, fewer cancer patients presented to acute services in total, driven mainly by a reduction in complications from cancer treatment and the disease itself. Nationally in the UK it is thought that 6,000 fewer cancer patients than expected received chemotherapy since the start of lockdown in March due to guidance from NHS England about prioritising patients. ${ }^{9}$ In line with this, Barts Cancer Centre reduced the number of systemic anticancer therapies (SACTs) administered by $20 \%$. This is likely to account for the fall in patients presenting with a complication of their cancer treatment. However, it does not explain why fewer patients presented with complications from their cancer. This may be due to patients' reluctance to attend hospitals in general, as shown by the reduction in attendances to the ED compared to previous years, and therefore increases the need for remote reviews and COVID-19-'free' areas where specialist oncology face-to-face reviews can occur. The chemotherapy hotline, in conjunction with increased CAAU capacity, is one way in which Barts Health have moved towards this goal.

There was a higher proportion of acute presentations due to a new cancer diagnosis in 2020, suggesting an impact on cancer referrals. Nationally, it has been reported that 290,000 fewer patients were referred to the urgent suspected cancer ' 2 -week wait' pathway since lockdown began. ${ }^{9}$ This reduction is thought to be due to fewer people going to their GP with cancer symptoms as well as reduced access to diagnostic pathways in the hospital as staff were redeployed to manage patients unwell with COVID-19. As such, our data reflect a higher proportion of new cancer patients presenting acutely unwell via the ED rather than through standard 2-week wait pathways during the COVID-19 pandemic, which may have implications for the cancer stage and survival. Independent of disruption to normal cancer referral pathways, new cancer patients presenting via the ED are known to have worse survival outcomes compared to those presenting via a nonemergency route. This is likely due to complex aetiologies including more advanced disease, older age and higher socioeconomic deprivation ${ }^{10,11}$ but we must also now ensure COVID does not play a role in increasing and contributing to these emergency presentations and negative effects on survival outcomes. Thus, we believe maintaining a robust AOS and educating patients on how to best seek the correct cancer services will be key in ensuring best management and in turn better cancer patient outcomes during future pandemics.

\section{Conclusions}

Based on these data, we suggest that NHS trusts consider restructuring their cancer services and AOS for future pandemics. We believe the '2-week wait' pathways should be maintained where possible to reduce the burden of new cancer diagnoses presenting via the ED. For those patients with known cancer or on SACT, consideration should be given to diverting these patients directly to oncology specialist centres and therefore away from the ED. We believe telephone triage advice for cancer patients and continued

Table 3. The recommended review location for patients after contacting the chemotherapy telephone hotline (mean number of patients per day)

\begin{tabular}{llllll} 
& $\begin{array}{l}\text { Telephone advice } \\
\text { only }\end{array}$ & $\begin{array}{l}\text { Advised to attend } \\
\text { ED }\end{array}$ & $\begin{array}{l}\text { Advised to attend } \\
\text { GP }\end{array}$ & $\begin{array}{l}\text { Advised to attend } \\
\text { CAAU }\end{array}$ & $\begin{array}{l}\text { Admission to } \\
\text { oncology ward }\end{array}$ \\
April 2019 & 5.7 & 3.8 & 1.4 & 0.4 & 0.0 \\
April 2020 & 7.8 & 1.5 & 0.9 & 1.4 & 0.5 \\
\% change from April 2019 & +36.8 & -60.5 & -35.7 & +250 & N/A \\
\hline CAAU = cancer acute assessment unit; ED = emergency department; N/A = not applicable.
\end{tabular}


remote AOS support is vital during any future COVID-19 pandemic, in order to support cancer patients navigating the need for acute medical support.

\section{Acknowledgements}

We would like to thank Heather Howton, Jennifer Nsowah and Emily Wang for their contributions to the AOS database.

\section{References}

1 Kuderer NM, Choueiri TK, Shah DP et al. Clinical impact of COVID-19 on patients with cancer (CCC19): a cohort study. Lancet 2020;395:1907-18.

2 Lee LYW, Cazier JB, Starkey T et al. COVID-19 mortality in patients with cancer on chemotherapy or other anticancer treatments: a prospective cohort study. Lancet 2020;395:1919-26.

3 Johnson B. Prime Minister's statement on coronavirus (COVID-19): 23 March 2020. www.gov.uk/government/speeches/pm-addressto-the-nation-on-coronavirus-23-march-2020 [Accessed 1 August 2020].

4 Sud A, Torr B, Jones ME et al. Effect of delays in the 2-week-wait cancer referral pathway during the COVID-19 pandemic on cancer survival in the UK: a modelling study. Lancet Oncol 2020;21:1035-44.

5 The National Confidential Enquiry into Patient Outcomes and Death. For better, or worse? A review of the care of patients who died within 30 days of receiving systemic anti-cancer therapy. NCEPOD, 2008. Available from www.ncepod.org.uk/2008sact.html.

6 National Chemotherapy Advisory Group. Chemotherapy services in England: ensuring quality and safety. NCAG, 2009.

7 Royal College of Physicians. Acute care toolkit 7: Acute oncology on the acute medical unit. RCP, 2015. Available from www.rcplondon. ac.uk/guidelines-policy/acute-care-toolkit-7-acute-oncology-acutemedical-unit.

8 Pinato DJ, Zambelli A, Aguilar-Company ] et al. Clinical portrait of the SARS-CoV-2 epidemic in European cancer patients. Cancer Discov 2020:10:1465-74.

9 Cancer Research UK. Over 2 million people waiting for cancer screening, tests and treatments. CRUK, 2020. https://scienceblog. cancerresearchuk.org/2020/06/01/impact-of-coronavirus-oncancer-services-revealed-over-2-million-people-waiting-forscreening-tests-and-treatments [Accessed 1 August 2020].

10 McPhail S, Elliss-Brookes L, Shelton J et al. Emergency presentation of cancer and short-term mortality. Br ] Cancer 2013;109:2027-34.

11 Abel GA, Shelton J, Johnson S et al. Cancer-specific variation in emergency presentation by sex, age and deprivation across 27 common and rarer cancers. Br J Cancer 2015;112:S129-36.

Address for correspondence: Dr Shanthini Crusz, Department of Medical Oncology, Basement, King George V Building, St Bartholomew's Hospital, London EC1A 7BE, UK.

Email: shanthini.crusz@nhs.net 